\title{
Numerical Analysis and Experimental Study of a New Y-block Chuan-Jun $\mathrm{LI}^{1, \mathrm{a}}$, Wan-Wan HUANG ${ }^{1, b, *}$, Zhi-Qiang WANG ${ }^{2, b}$, Kai $\mathrm{LI}^{1, \mathrm{~b}}$, Chuan-Gang LIU ${ }^{3, \mathrm{~b}}$ \\ ${ }^{1}$ Jiangsu University, Zhenjiang Jiangsu 212013, China \\ ${ }^{2}$ China Federation of machinery industry, Beijing 100823, China \\ ${ }^{3}$ CNOOC (China) Co., Ltd. Tianjin Branch, Tianjin 300452, China \\ aluckyjourney@gmail.com, bjsdxhww@126.com \\ ${ }^{*}$ Corresponding author
}

Keywords: Hydraulic Control, Thermal Recovery of Heavy Oil, Standard K- $\varepsilon$ Model, Numerical Simulation, Energy Consumption.

\begin{abstract}
A new Y-block design's function and hydraulic performance were verified through numerical methods and experimental validation. The velocity fields of its internal flow channel and turbulence energy under different opening levels were analyzed by ANSYS. Combined with experiments, the automatic switching function of the Y-block was examined. Based on the hydraulic control theory, the new structure of Y-block was designed by characters of one-way valve pressure of forward and reverse. A three-dimension model was set up by Pro-E, and the velocity and energy consumption distribution of one-way valve at different opening levels were shown with standard K- $\varepsilon$ of ANSYS. Combined with the test data, the max energy consumption of the new configuration was achieved and its self-control ability was proved. The results show that the max energy consumption of the new Y-block is lower $0.5 \mathrm{Mpa}$; its self-control ability can meet the requirement of the thermal recovery of heavy oil in the switching action.
\end{abstract}

\section{Introduction}

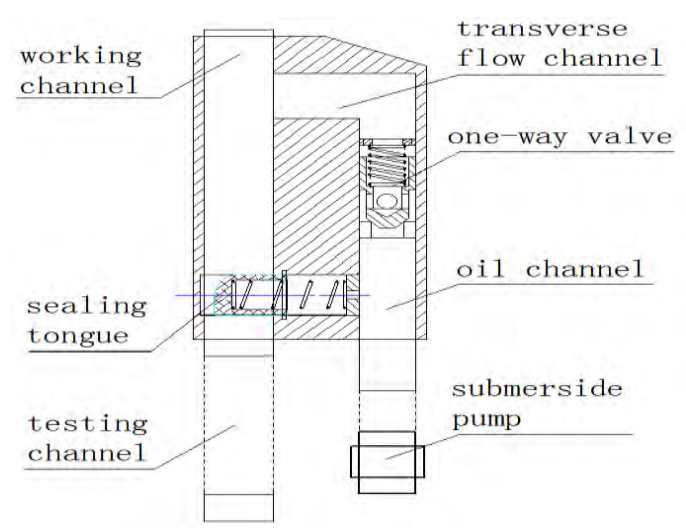

Fig. 1 Structure Schematic

High-temperature thermal recovery $[1,2,3]$ is usually carried out more than $1000 \mathrm{~m}$ below sea level The oil pressure, temperature, moisture and other parameters would change with the mining operations which would affect the rationality of the crude oil exploitation directly. The relevant sensors are put into the bottom of the production wells to measure and control promptly the undersea reservoir parameters. The production string is necessary to convert from the oil status to the testing status. Y-block is the device to achieve the function. At present, the Sams joint $[4,5]$ is widely used in the thermal recovery for its simple structure and easy operation. However, it needs manual operation frequently to achieve the transform of two states. Many scholars have developed a variety of new intelligent adapters $[6,7,8,9]$ based on practical needs. However, the designed equipment cannot be put into practical application for its inadequate availability and reliability. In order to solve these 
problems, a new type of intelligent conversion Y-block (Fig.1) is proposed. The reliability of intelligent conversion function and energy dissipation of the device is studied by experiment and numerical analysis method. Internal flow velocity distribution diagram and turbulent kinetic energy dissipation of different opening degree can be achieved. The maximum power consumption of the new device is analyzed to verity that the energy loss whether meets the demands based on the velocity distribution and turbulent kinetic energy dissipation cloud at different degrees of the flow field. An experimental platform is built on the processing device, and prototype verifies the self-switching operation which provides powerful reference for the device being put into practical application.

\section{Structural Features and Working Principle}

\section{Structural Features}

Structure of The new intelligent conversion Y-block is shown in Fig.1, which is mainly composed of joint body, sealing tongue, the sealing channel and one-way valve. Testing channel, oil channel and working channel are arranged in the main body of production. Using the opening and closing of one-way valve to form a reverse pressure between two compression face of sealing tongue, then to control the sealing tongue to slide to the left (testing channel is closed and oil channel is opened) or the tight (testing channel is opened and oil channel is closed), so as to realize the automatic switching between oil status and testing status. Design of the whole device must be applied to the operation environment of $9-5 / 8$ production pipe, and energy consumption should be limited in $0.5 \mathrm{MPa}$.

\section{Working Principle}

Device uses the one-way valve and the seal sliding to realize the test channel opening so as to achieve automatically switching between the oil status and testing status. Then it is not necessary to worry about the inner loop caused by the connectivity between the oil channel and the testing channel, nor the pressure relieve problem of testing channel when the pump stops. The specific working principle is stated as follows:

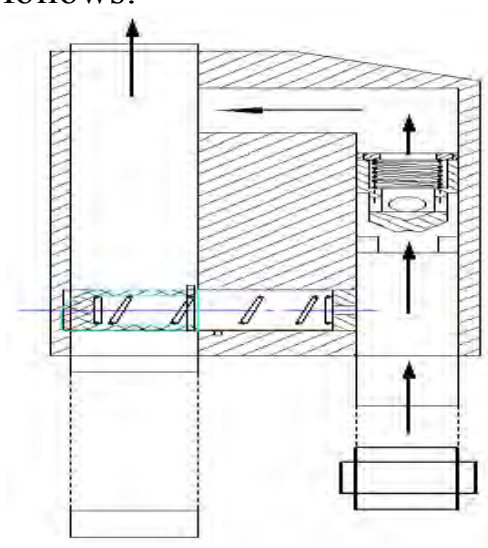

Fig. 2 Oil Production Status

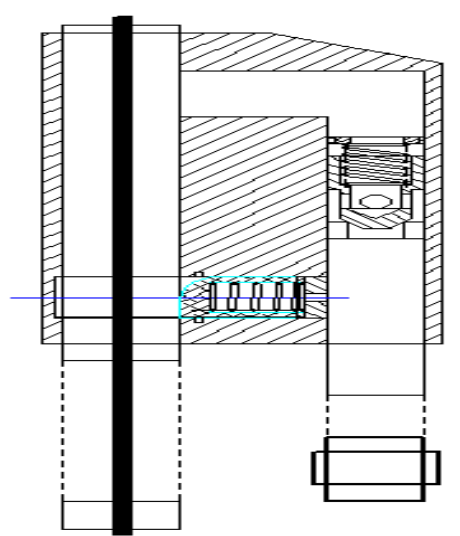

Fig. 3 Testing Status

(a) Oil status (shown in Fig.2). The crude oil overcomes the valve opening pressure of the one-way valve and then flows to the working channel. Submersible pump operation and sealing tongue will realize the sealing of testing channel under the pressure. The crude oil overcomes the valve opening pressure of the one-way valve and then flows to the working channel. The inlet pressure of oil channel is set as $10 \mathrm{MPa}$ and flow is $60 \mathrm{~m} 3 /$ day.

(b) Testing status (shown in Fig.3). Submersible pump is closed. The oil pressure of the working channel about $10 \mathrm{MPa}$ is formed under the action of gravity. Sealing tongue can idiomatically reset under the pressure difference so that the testing channel is opened through up and down, so as to ensure the safety relief of pressure. Then it is time to put the test instrument pass the test channel into the bottom of the oil well for testing work. 


\section{Numerical Simulation}

\section{Pre-processing}

According to the structure parameters of the new joint design, three-dimensional modeling of the equipment under oil status is constructed by Pro/E software. Then the model is imported to ANSYS ICEM software to make hexahedral mesh division, doing encryption processing at variable flow channel cross-sectional diameter and turning parts. Making analysis of mesh independence analysis, it is funded mesh number 893410 is suitable for the model finally. It is suitable to approximate reflect the basic characteristics of the internal flow field by means of the unsteady flow and in-compressible flow. The standard K- $\varepsilon$ turbulence model in CFX software is used to simulate the internal flow field under 4 different openings of one-way valve at high temperature based on the precondition of satisfying the no slip wall condition under the condition of high temperature of $300^{\circ} \mathrm{C}$ and inlet pressure $10 \mathrm{MPa}$.

\section{Simulation Analysis}

The simulation of one-way valve is in 4 different openings $(10 \mathrm{~mm}, 24 \mathrm{~mm}, 38 \mathrm{~mm}, 51 \mathrm{~mm})$ under the steady flow field were carried out. Velocity variation and energy change of the main parameters of the interior flow were shown in Fig. 4 and Fig. 5.

Fig. 4 shows the velocity distribution diagram of total pressure under 4 different openings of one-way vale. We can learn from the diagram crude oil sprays from valve port via one-way valve, and flow separation phenomenon will occur in the valve outlet. In the opening states of 1, 2, 3, vortex appears in the valve cavity respectively and consuming mainstream energy in motion which will cause the pressure drop and energy reduction. In addition, a cone jet fluid formation is presented through a one-way valve flow to the transverse pipe of fluid flow. Jet velocity increases with the one-way valve opening degrees, thus result in great impact on the flow pipe of transverse flow channel. According to the energy equation of internal flow, the one-way valve openings in different degree have different influence on flow channel pressure. Large pressure drop is formatted in the center of the vortex in the rear area of valve and valve outlet. The maximum of pressure drop up to $0.038 \mathrm{MPa}$ under the opening of 3 . It is in the range of pressure drop designed, so meeting the requirements.

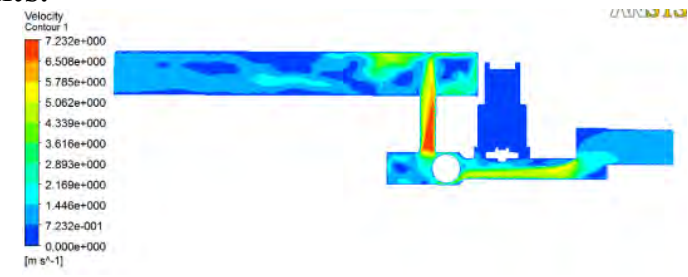

(a) Opening 1 of one-way valve

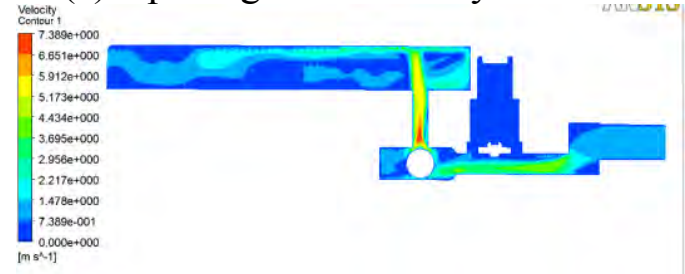

(c) Opening 3 of one-way valve

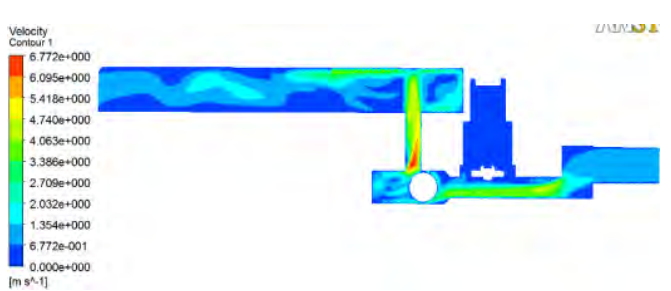

(b) Opening 2 of one-way valve



(d) Opening 4 of one-way valve

Fig. 4 Velocity Distribution Diagram

Fig. 5 shows the distribution map of global turbulence kinetic energy under 4 different openings of one-way vale. It can be obtained that kinetic energy of turbulence under small valve opening1 is maximum. Besides the energy loss is mainly concentrated on the valve inlet. With the valve opening increases, the maximum turbulent kinetic energy gradually transfers to transverse flow channel. However, turbulent kinetic energy of vortex center area decreases, accompanied with the low pressure area. It indicates that with the increase of the valve opening, the impact loss of horizontal liquid flow pipeline gradually increases. 




(a) Opening 1 of one-way valve

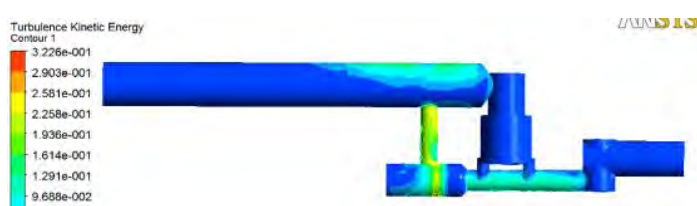

(b) Opening 2 of one-way valve

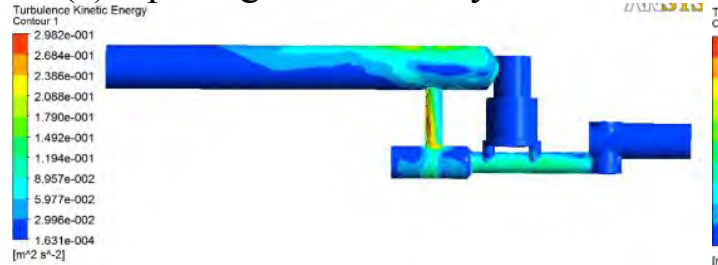

(c) Opening 3 of one-way valve

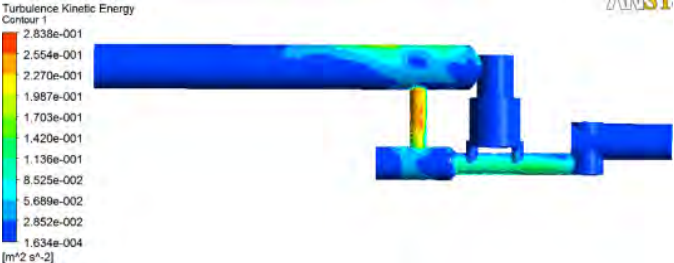

(d) Opening 4 of one-way valve

Fig. 5 Distribution Map of Global Turbulence Kinetic Energy

\section{Structure Optimization}

From the simulation analysis of flow field in different openings of the one-way valve, it can be found that the violent disturbance phenomenon occurs when the fluid flows through the ball valve to one-way valve outlet, Since the fluid is influenced by flow resistance and the mutation caused by one-way valve. Vortex is formed in this proceeding, so greater pressure loss is caused.

\section{Functional Verification Experiments}

Although the device can meet the application requirements and can work in high temperature and high pressure conditions in theory, whether the accurate intelligent transformation can be achieved between the two states and whether it can meet the sealing requirement still need experiment to verify.

\section{Experimental Scheme}

The experimental device designed was shown in Fig. 6, including high pressure plunger pump, regulating valve, one-way valve, water tank and a corresponding pipelines. The ideas of operation were described as follows:

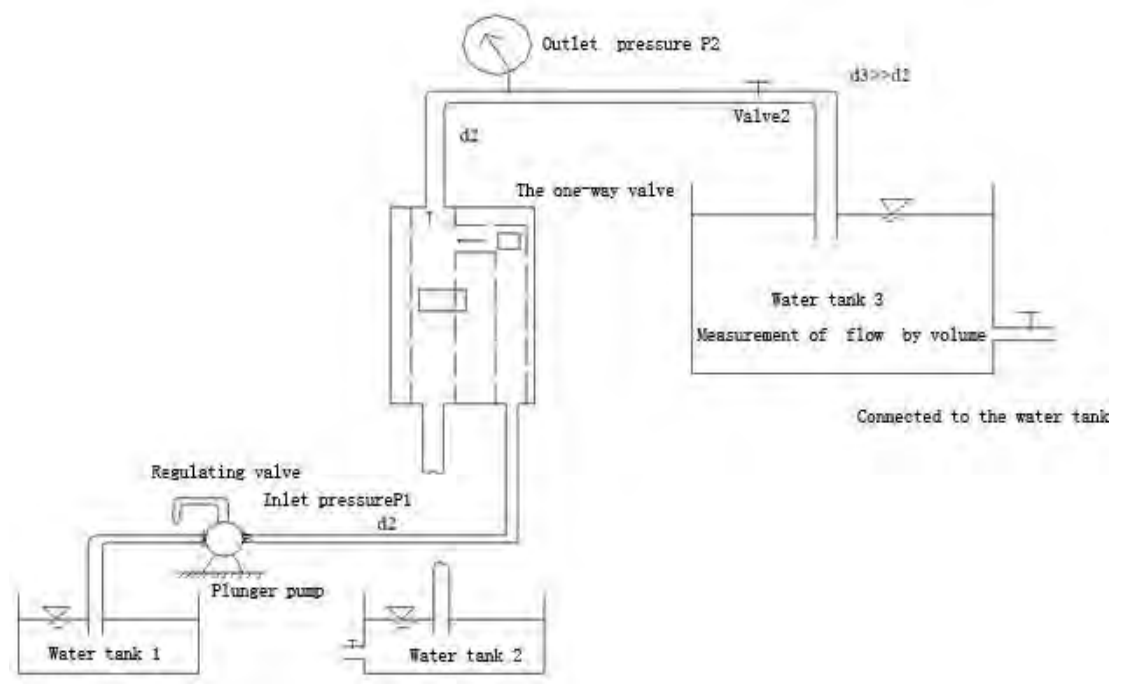

Fig. 6 Schematic Diagram of the Experimental Scheme

(1)Opening the power of plunger pump (it can provide maximum pressure $25 \mathrm{MPa}$ ), so it can provide the opening pressure to the device. Adjusting the flow through the pump and regulation valve at the joint is used for the qualitative control of pressure in the pump oil channel. By observing lower 
part of the test channel (corresponding to the water tank 2) whether has medium overflowing from sealing tongue, the sealing effect can be verified. The relationship between the outlet flow $\mathrm{Q}$ obtained by volume measurement and imported pressure P1 can be achieved. At the same time, observing the upper test channel whether has medium flowing out continuously to determine whether the joint designed meets the requirements of production state.

(2)Verification of testing state: connecting piston pump with the upper working channel firstly. Using the maximum opening pressure provided by a plunger pump to replace the static pressure generated by oil column of 1200 meters to observe the action of sealing tongue. The aim is to observe whether sealing tongue can home to seal groove completely in the role of hydrostatic pressure or not, so as to ensure the opening of test channel. The data obtained from the repeat experiment was shown in tab.1.

Tab.1 The Opening Pressure Data of Sealing Tongue

\begin{tabular}{|c|c|c|c|c|c|}
\hline The number of measurements & 1 & 2 & 3 & 4 & 5 \\
\hline $\begin{array}{c}\text { The opening pressure data of seal- } \\
\text { ing tongue P(Mpa) }\end{array}$ & 0.42 & 0.55 & 0.5 & 0.52 & 0.48 \\
\hline
\end{tabular}

\section{The Data Processing of Experiment}

According to the recording data from experimental process, make processing conversion and the analysis induction, then three curves are summarized as following. The relation curve between outlet flow Q1 and imported pressure P1 is shown in Fig.7. The relation curve between the flow leakage Q2 and the import pressure P1 is shown in Fig.8. The relation curve between pressure difference of the inlet and outlet and inlet pressure is shown in Fig.9.

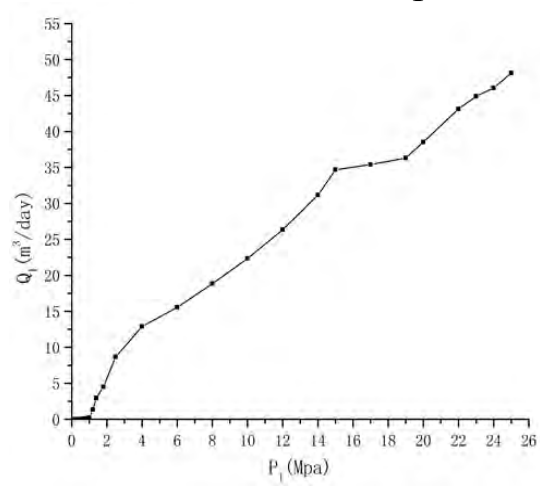

Fig. 7 Relation Curve between Outlet Flow Q1 and Imported Pressure P1

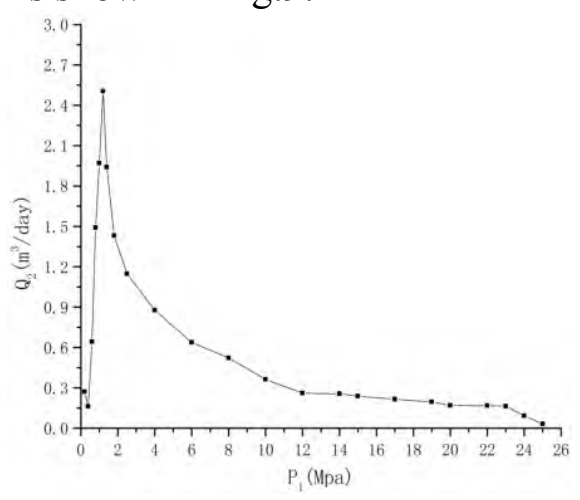

Fig. 8 Relation Curve between the Flow Leakage Q2 and the Import Pressure P1

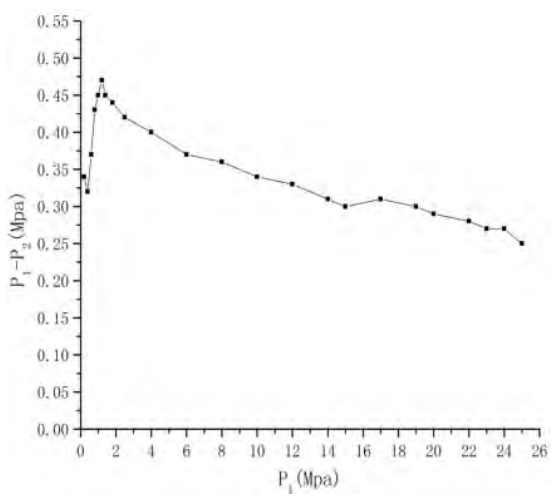

Fig. 9 Relation Curve between Pressure Difference of the Inlet and Outlet Pressure

\section{Results of Experiment}

(1) As is shown in fig.7, outlet flow $Q_{1}$ is increased with the increase of inlet pressure $P_{1}$. When $P_{1}$ is up to $0.5 \mathrm{M} \mathrm{Pa}$, one-way valve is opened. When $\mathrm{P}_{1}$ is up to $1.25 \mathrm{M}$ pa, one-way valve is fully opened. Outlet flow and inlet pressure approximately changes in accordance with the direct ratio relationship. After conversion with volume method, the biggest export flow can reach up to $48.5 \mathrm{~m}^{3} /$ day under the pressure of $25 \mathrm{M}$ pa, which reflects that flow deviation is little compared with production requirements.

(2) As is shown in fig.8, with the increase of inlet pressure $\mathrm{P}_{1}$, micro leakage turns up underneath the test channel. Leakage reaches peak $2.55 \mathrm{~m}^{3} /$ day when $\mathrm{P}_{1}$ reaches around $1.25 \mathrm{M} \mathrm{Pa}$. However, with further increase of pressure, leakage is falling and close to zero. It shows that the sealing tongue can seal the test channel at about $1.5 \mathrm{M} \mathrm{Pa}$, but the sealing effect is increased with the increase of inlet pressure.

(3) As is shown in fig.9, pressure differential exists between import and export due to micro leakage of the equipment. The pressure loss reaches peak $0.46 \mathrm{M}$ pa when $\mathrm{P}_{1}$ is up to1.25 $\mathrm{M} \mathrm{Pa}$, which is limited in the range of production license. 
(4) As is shown in tab.1, testing channel can be opened under the pressure $0.5 \mathrm{M}$ pa, which can realize safety pressure relief at the test status. It reflects that sealing tongue can replace smoothly under low pressure.

\section{Conclusion}

(1)According to the pressure distribution, velocity distribution and turbulent dissipation nephogram of the internal flow field at different opening, it can be obtained that the pressure loss and turbulent kinetic energy loss of the device are in the design range, so the joint can meet the requirements of application under high temperature and high pressure.

(2)The numerical simulation can determine the position of the maximum turbulent kinetic energy dissipation. It was found in the fluid disturbed flow area of one-way valve, which made contribution to the optimization of the structure of device in the aspect of reducing the energy loss.

(3)It can realize the sealing of test channel at the oil production state and the opening of test channel at testing state by the way of hydraulic control to control one-way valve and the pressure of the sealing tongue on both sides, which makes the function of the joint verified.

\section{Acknowledgment}

The authors wish to thank the 12th Five Year Plan of national major projects of science and technology (2011ZX05024-005-004), the 12th college student scientific research project of Jiangsu University (Y12A155) for the financial support.

\section{References}

[1]Defu Che, Huixiong Li. Multi-phase flow and its application. Xi'an: Xi'an Jiao Tong University press, 2006:17-19.

[2]Xu Wang. Heavy oil recovery techniques in Liao he oilfield and key technical research in next step. Petroleum Exploration and Development, 2006, 4th period.

[3]Yigang Wang, Zhiyong Wang, Dong Meng. Patent technology status of China's petroleum exploitation equipment and suggestions of development. CHINA INVENTION \& PATEN, 2012, 2nd period.

[4]Rosilda Maria Gomes de Lima, Glória egina da Silva Wildhagen, José Waldemar Silva Dias da Cunha. Removal of ammonium ion from produced waters in petroleum offshore exploitation by a batch single-stage electrolytic process. Journal of Hazardous Materials, 2009, Pages 1560-1564.

[5]Mingren Shao, Guichuan Li, Shushan Guo, Xiaocheng Zhang . Integrated "Y" joint utility model. China Offshore Oil Corporation, 2008.

[6]Jin Yang, Liping Yang, Jie Su. Well spring type Y connector. China Offshore Oil Corporation, 2005.

[7]Shujie Liu, Jin Yang. A self-switching type "Y" joint. China Offshore Oil Corporation, 2008.

[8]JinmingWang. Optimization design of Oil well intelligent " $\mathrm{Y}$ " joint. China University of Petroleum, 2008.

[9]Rosilda Maria Gomes de Lima, Glória egina da Silva Wildhagen, José Waldemar Silva Dias da Cunha. Removal of ammonium ion from produced waters in petroleum offshore exploitation by a batch single-stage electrolytic process. Journal of Hazardous Materials, 2009, Pages 1560-1564.

[10]Bingbing Ji, Jinping Chen. ANSYS ICEM CFDANSYS ICEM CFD example explanation of mesh division. Chinese water conservancy and Hydropower Press, first edition, January 1, 2012. 